\title{
Material-Independent and Size-Independent Tractor Beams for Dipole Objects
}

\author{
Novitsky, Andrey; Qiu, Cheng-Wei; Lavrinenko, Andrei
}

Published in:

Physical Review Letters

Link to article, DOI:

10.1103/PhysRevLett.109.023902

Publication date:

2012

Document Version

Publisher's PDF, also known as Version of record

Link back to DTU Orbit

Citation (APA):

Novitsky, A., Qiu, C-W., \& Lavrinenko, A. (2012). Material-Independent and Size-Independent Tractor Beams for Dipole Objects. Physical Review Letters, 109(2), 023902. https://doi.org/10.1103/PhysRevLett.109.023902

\section{General rights}

Copyright and moral rights for the publications made accessible in the public portal are retained by the authors and/or other copyright owners and it is a condition of accessing publications that users recognise and abide by the legal requirements associated with these rights.

- Users may download and print one copy of any publication from the public portal for the purpose of private study or research.

- You may not further distribute the material or use it for any profit-making activity or commercial gain

- You may freely distribute the URL identifying the publication in the public portal

If you believe that this document breaches copyright please contact us providing details, and we will remove access to the work immediately and investigate your claim. 


\title{
Material-Independent and Size-Independent Tractor Beams for Dipole Objects
}

\author{
Andrey Novitsky, ${ }^{1}$ Cheng-Wei Qiu, ${ }^{2, *}$ and Andrei Lavrinenko ${ }^{1}$ \\ ${ }^{1}$ DTU Fotonik, Department of Photonics Engineering, Technical University of Denmark, \\ $\emptyset$ rsteds plads 343, DK-2800 Kongens Lyngby, Denmark \\ ${ }^{2}$ Department of Electrical and Computer Engineering, National University of Singapore, \\ 4 Engineering Drive 3, Singapore 117576, Singapore \\ (Received 2 April 2012; published 12 July 2012)
}

\begin{abstract}
A Bessel beam without an axial gradient can exert a pulling force on an object [A. Novitsky, C. W. Qiu, and H. Wang, Phys. Rev. Lett. 107, 203601 (2011)]. However, it cannot be called a "tractor beam" per se, as long as the light pulling effect is ultrasensitive to the object's material and size, a perturbation of which will make the optical traction go away. In this Letter, we investigate and report on the universality for a Bessel beam to be either a material-independent or size-independent optical tractor beam within the dipolar regime. Moreover, a general condition for a nonparaxial laser to be simultaneously a material- and size-independent tractor beam is proposed. These universal pulling effects and conditions are discussed in association with insight on modified far-field scattering, scattering resonances, and induced polarizabilities. Interestingly, we find that the acoustic pulling force exhibits only size independence, owing to the acoustic scattering theory in contrast to the light scattering counterpart. The findings pave the way for the realistic engineering and application of universal tractor beams pulling a wide variety of objects.
\end{abstract}

DOI: 10.1103/PhysRevLett.109.023902

PACS numbers: 42.15.Dp, 37.10.Jk, 42.50.Wk

When one studies the pulling effect of light, it is natural to consider optical trapping [1] or tweezers [2], which can drag particles due to gradient fields. An optomechanical system can be adopted to form the field gradient between a waveguide and a microdisk, leading to the reactive force [3]. The mechanism of pulling by optical tweezers owes itself to the gradient intensity, resulting in the force driving the object to the point of the field extremum. The field extremum can be formed by converging a plane wave with a lens or interfering a number of different waves. However, such a pulling effect depends on the location of the field extremum rather than the light source, and the driving force is not due to the negative radiation pressure.

It is possible to overcome this restriction by interfering two different nondiffracting Bessel beams [4]. The pulling force by this approach relies on a highly lossy particle and high-index background material whose refractive index is higher than that of the particle, which is impractical. It is also asserted that only the interference of two (or more) different Bessel beams could result in the negative optical force [4]. However, this assertion is not rigorous because the negative optical force can be achieved by a single nonparaxial Bessel beam, due to the interference of electric and magnetic dipoles or higher-order multipoles or by a single static solenoid beam [5]. There are some ubiquitous proofs of pulling forces found in thermodynamics [6], optics [7], and acoustics [8].

A single paraxial nondiffracting wave (e.g., a Bessel beam [9]) results in pushing objects along the light propagation path. Such nondiffracting beams can also trap particles in its cross section due to the transverse field gradient $[1,2]$. However, the pulling is forbidden by momentum conservation $[10,11]$ and, thus, cannot be achieved by a single paraxial nondiffracting light.

Recent works on nonparaxial light beams $[7,12]$ have suggested that a single beam can be a tractor beam, but in order to show that a pulling force is possible, one needs to wisely select the numerical aperture of the beam (nonparaxiality of the beam) and the particle's permittivity and size. In other words, if a subtle deviation in one of those parameters spoils the phenomenon of traction, the optical traction is neither stable nor universal. This problem has not been answered or attempted in the pioneering work on tractor beams, which basically showed some typical cases for the optical force being negative. Alternatively, a series of beams with individual control of polarization and phase of each wave can exert a negative force on a collection of particles treated as a black box [13]. That method is robust because those multiple incident waves provide a large degree of freedom for optimization of all magnitudes and phases. The price for robustness is that the optimized phases need sophisticated control in practice, and all incident waves require reoptimization for different targets, sizes, or distributions. These problems become critical in practical scenarios. In the mean time, the origin of the negative optical force has not been answered previously. Thus, it is imperative to answer and solve the following problems at once: (i) What is the most fundamental reason for the pulling force and how to interpret the negative force value by electromagnetic (EM) language rather than by mathematical value? (ii) Can the pulling force be independent (at least quasi-independent) of an object's material or size? (iii) Can a quantitative condition be proposed for achieving the utmost independence on 
material and size? (iv) Can the universality condition be scaled to acoustics or even more general disciplines for the pulling force?

Based on those important motivations, we demonstrate the single-independence (on either material or size) and double-independence (on both material and size) tractor beams for dipolar objects. We derive the necessary condition for such independent pulling forces and investigate the acoustic counterpart where only size dependence is found. Last but not least, the fundamental physics of a tractor beam, its independence on dipolar objects, and its relation to EM scattering are discussed and explained in the exact context of classical electrodynamics. It opens up an unprecedented way of achieving optical traction forces in practice stably and universally. In the dipole approximation, we deal only with electric and magnetic dipole moments. Assume a spherical bead of radius $R$ is placed in vacuum and characterized by both dielectric permittivity $\varepsilon$ and magnetic permeability $\mu$. Polarizabilities of the bead are expressed in terms of the Mie coefficients [14]

$$
\begin{aligned}
& a_{\nu}=\frac{n^{2} j_{\nu}(n x)\left(x j_{\nu}(x)\right)^{\prime}-\mu j_{\nu}(x)\left(n x j_{\nu}(n x)\right)^{\prime}}{n^{2} j_{\nu}(n x)\left(x h_{\nu}^{(1)}(x)\right)^{\prime}-\mu h_{\nu}^{(1)}(x)\left(n x j_{\nu}(n x)\right)^{\prime}}, \\
& b_{\nu}=\frac{\mu j_{\nu}(n x)\left(x j_{\nu}(x)\right)^{\prime}-j_{\nu}(x)\left(n x j_{\nu}(n x)\right)^{\prime}}{\mu j_{\nu}(n x)\left(x h_{\nu}^{(1)}(x)\right)^{\prime}-h_{\nu}^{(1)}(x)\left(n x j_{\nu}(n x)\right)^{\prime}},
\end{aligned}
$$

where $x=k_{0} R, k_{0}=\omega / c$ is the wave number in vacuum, $\omega$ is the angular frequency, $c$ is the speed of light, $n=\sqrt{\varepsilon \mu}$ is the bead's refractive index, $j_{\nu}$ and $h_{\nu}^{(1)}$ are the spherical Bessel and Hankel functions of the order $\nu$, prime means derivative with respect to the argument, e.g., $\left(n x j_{\nu}(n x)\right)^{\prime}=$ $d\left(n x j_{\nu}(n x)\right) / d(n x)$. The sum of all harmonics (dipole, $\nu=1$; quadrupole $\nu=2$, etc.) results in the scattered field produced by the illuminated particle. The dipole particle has strong dipole terms $a_{1}$ and $b_{1}$ in the scattering and can be characterized by the electric $\alpha_{e}=3 i a_{1} /\left(2 k^{3}\right)$ and magnetic $\alpha_{m}=3 i b_{1} /\left(2 k^{3}\right)$ polarizabilities.

Such dipole approximations are valid when the quadrupole (and higher) terms are weak $\left(\left|a_{2}\right|,\left|b_{2}\right| \ll\left|a_{1}\right|,\left|b_{1}\right|\right)$. We quantify this criteria by applying the rule $\left|a_{2}\right|<$ $0.2\left|a_{1}\right|$. This criterion confines the region of interest within the unshaded domain in Figs. 1(a) and 1(b). We expect intensive interaction between dipole moments in a region of intermediate size parameters $k_{0} R \sim 1$, where both Mie coefficients $a_{1}$ and $b_{1}$ are large.

Since many materials lose magnetism at high frequencies or do not possess a strong magnetic response in general, it would be beneficial to operate with nonmagnetic materials instead. A magnetic dipole moment indeed can artificially be induced in a large particle [15], and this is confirmed by large $b_{1}$ (therefore, magnetic polarizability $\alpha_{m}$ ) for the nonmagnetic spheres shown in Fig. 1(b). Thus, we start with nonmagnetic objects and come back to magnetic particles later.
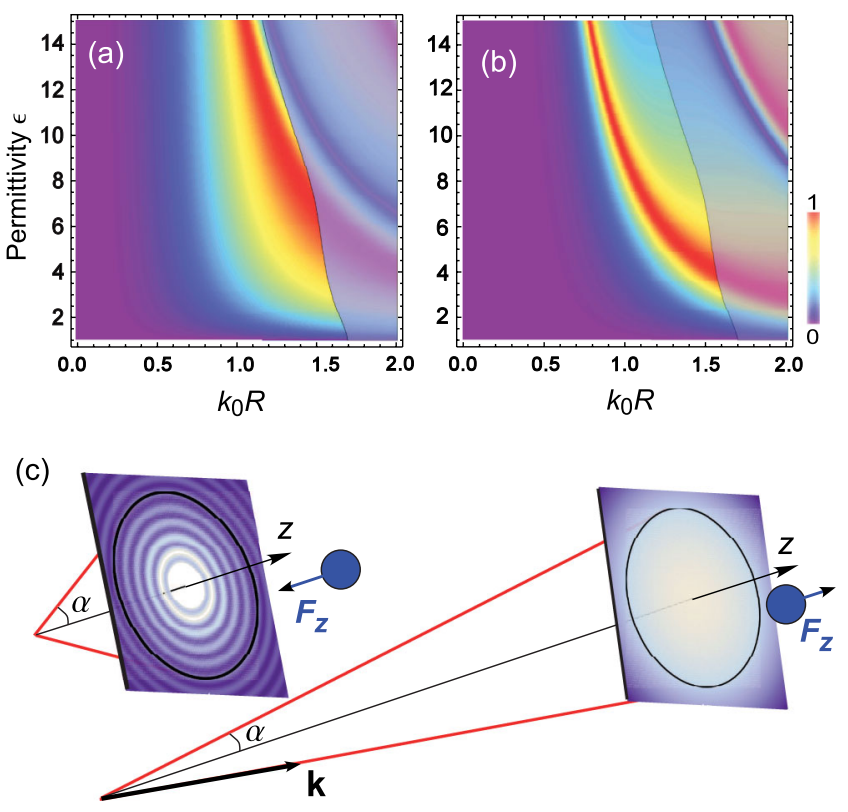

FIG. 1 (color online). Mie coefficients (a) $a_{1}$ and (b) $b_{1}$ for the nonmagnetic $(\mu=1)$ spherical particle. On the right-hand side of the figures, the region of invalid dipole approximation $\left(\left|a_{2}\right|>\right.$ $\left.0.2\left|a_{1}\right|\right)$ is shaded. (c) Pulling force by a nonparaxial beam ( $\alpha$ is large) versus pushing force for paraxial beam ( $\alpha$ is small).

Let us consider a nonmagnetic sphere of dielectric permittivity $\varepsilon$ in vacuum illuminated by a single nondiffracting Bessel beam $\{\mathbf{E}(\mathbf{r}, t), \mathbf{H}(\mathbf{r}, t)\}=\exp \left(i k_{z} z+\right.$ $i m \varphi-i \omega t)\{\mathbf{E}(r, \varphi), \mathbf{H}(r, \varphi)\}$, where $k_{z}$ is the longitudinal wave number, and $m$ is the beam order. Nonparaxiality $\left(k_{z}\right.$ is not close to $k_{0}$ ) of such Bessel beams is crucial for inducing the negative optical force upon the microscopic particle. Vector Bessel beams considered herein are the exact solutions of the Maxwell equations in cylindrical coordinates [16],

$\mathbf{E}(r, \varphi)=\left(J_{m}\left(q k_{0} r\right) c_{2} \mathbf{e}_{z}-\frac{1}{q} c_{1}\left(\mathbf{e}_{z} \times \mathbf{b}\right)+\frac{\beta}{q} c_{2} \mathbf{b}\right)$,

where $\mathbf{b}=i J_{m}^{\prime}\left(q k_{0} r\right) \mathbf{e}_{r}-\left(m / q k_{0} r\right) J_{m}\left(q k_{0} r\right) \mathbf{e}_{\varphi}$, and parameters $\beta$ and $q$ are defined below. Equation (2) describes the superposition of phase-shifted TE (complex amplitude $c_{1}$ ) and TM (amplitude $c_{2}$ ) Bessel beams. Wave vectors of all plane waves forming the light beam lie on the cone surface with angle $2 \alpha$ at the vertex of the cone [see Fig. 1(c)]. Then the nonparaxiality of the beam can be uniquely characterized by the cone angle $\alpha$. The longitudinal and transverse components of the wave vector are expressed as $k_{z}=k_{0} \beta=k_{0} \cos \alpha$ and $k_{\perp}=k_{0} q=k_{0} \sin \alpha$, respectively. The beam configuration follows the previously defined optimal parameter, i.e., $c_{2} / c_{1}=i$ (see the analysis of the influence of $c_{2} / c_{1}$ [7]).

The time-averaged force on a dipole particle can thus be calculated as [17] 


$$
\begin{aligned}
\left\langle F_{z}\right\rangle= & \frac{k_{0} \beta}{2}\left(\operatorname{Im}\left(\alpha_{e}\right)|\mathbf{E}|^{2}+\operatorname{Im}\left(\alpha_{m}\right)|\mathbf{H}|^{2}\right) \\
& -\frac{k_{0}^{4}}{3} \operatorname{Re}\left(\alpha_{e} \alpha_{m}^{*} P_{z}\right),
\end{aligned}
$$

where $P_{z}=\mathbf{e}_{z} \cdot\left(\mathbf{E} \times \mathbf{H}^{*}\right)$, and its real part is proportional to the $z$ component of the Poynting vector $S_{z}$. It should be noted that the dipole particles are considered in this paper, and their sizes are not much smaller than the wavelength. It can be observed from the second term in Eq. (3) that the axial force formula for the dipolar object as described in Eq. (3) is fundamentally distinguished from that for the Rayleigh particle in Ref. [7].

The first two terms in Eq. (3) describe the contributions of the electric and magnetic dipoles. For passive particles $\left[\operatorname{Im}\left(\alpha_{e, m}\right)>0\right]$, these contributions are always positive. The last term in Eq. (3) is responsible for the interaction between the dipoles, and the negative interaction term leads to the pulling force $\left\langle F_{z}\right\rangle<0$. It is obvious from Eq. (3) that the pulling-force effect requires small longitudinal wave numbers $k_{z}=k_{0} \beta$ (large cone angle $\alpha$ ) and large positive (forward-directed) Poynting vectors.

Equation (3) is applied for the computation of the optical force $\left\langle F_{z}\right\rangle$ as demonstrated in Fig. 2(a). The region corresponding to the dipole approximation is not shaded. It is true that such abrupt boundary is not realistic, but its functionality is confirmed by the comparison with the exact solution involving both the dipole and higher-order moments. The black curve in Fig. 2(a) for $\varepsilon=6$ ideally reproduces negative values of the optical force for $k_{0} R$ between 1 and 1.3. At the same time, in the shaded region further away from the boundary the discrepancy of the exact and dipole forces is significant because a consistent recognition of the quadrupole terms is required.

It is interesting to follow what exactly happens to the farfield scattering diagram when the optical pulling force is observed. In Fig. 2(a), the pulling force exhibits a recognizable feature in the far field (e.g., the backscattering is weak and forward scattering dominates) due to the increased momentum by redirecting photons from other directions toward the forward direction. Our scheme is in contrast to the amplified forward momentum using gain media [18], i.e., pumping more photons toward the forward direction. Usually the cone angle $\alpha$ is small for paraxial beams. In this case, the forwardly scattered field captured by the cone of $2 \alpha$ at the vertex is negligible compared to the backward scattering. The backward scattering pushes a particle in accordance with the momentum conservation. The same reasoning holds for the large cone angles (see the scattering diagram in Fig. 2 for $k_{0} R=0.7$ ). The interaction of the electric and magnetic dipoles can be manipulated to redirect the scattered field forward, while a large $\alpha$ allows the forward scattering to exceed over the backward scattering (see the scattering diagram for $k_{0} R=1.15$ ). Then, the momentum conservation dictates the appearance of the pulling optical force.
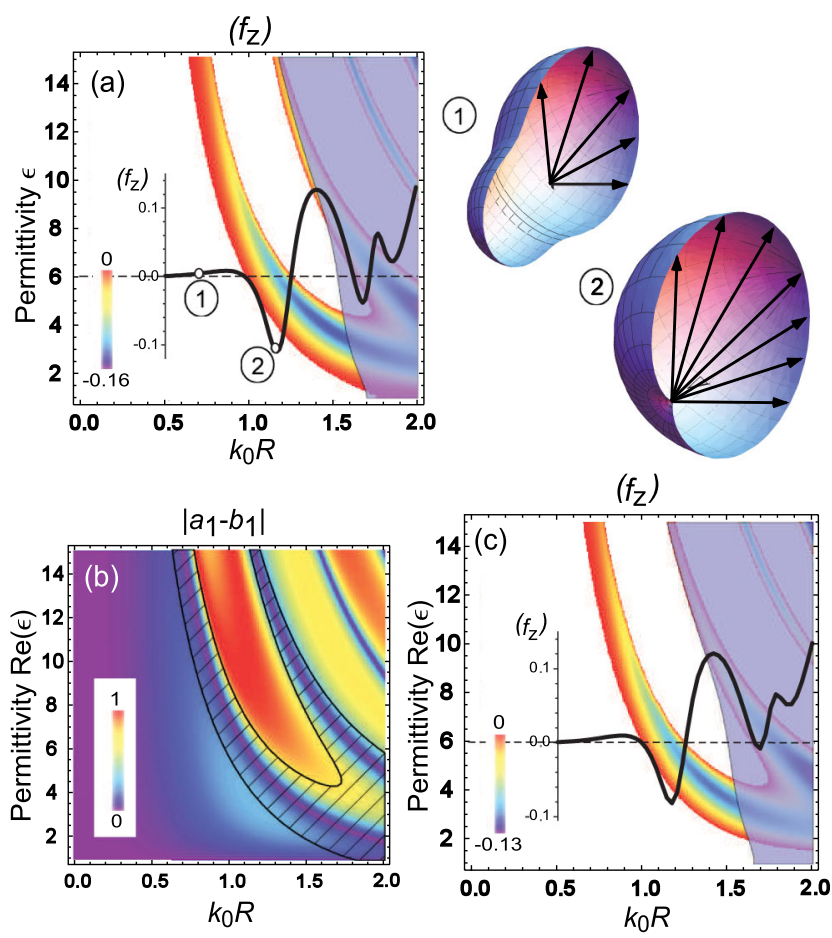

FIG. 2 (color online). Density plots demonstrate the dipole force $\left\langle f_{z}\right\rangle=\left\langle F_{z}\right\rangle k^{2} /\left|c_{1}\right|^{2}<0$ calculated according to Eq. (3) for (a) transparent and (c) absorptive $[\varepsilon=\operatorname{Re}(\varepsilon)+0.1 i]$ nonmagnetic spherical beads. Region beyond the dipole approximation is shaded. Black curves in (a) and (c) depict exact the optical forceop $\left\langle f_{z}\right\rangle$ at $\varepsilon=6$ and $\varepsilon=6+0.1 i$, respectively, when higher moments have been taken into account. Numbers 1 and 2 indicate scattered far field for pushing $\left(k_{0} R=0.7\right)$ and pulling $\left(k_{0} R=1.15\right)$ optical forces, respectively. Black arrows show the forward scattering limited by the cone angle $\alpha=70^{\circ}$. (b) Absolute value of the difference of the Mie coefficients $\left|a_{1}-b_{1}\right|$ demonstrates that the pulling force (shaded region) appears near $\alpha_{e}=\alpha_{m}$. The nonparaxial Bessel beam has characteristics $m=1, \alpha=70^{\circ}, c_{2} / c_{1}=i$.

It is important that the pulling force is feasible for the nonmagnetic dipole objects of arbitrary permittivities or sizes (or at least within a large range of variation). This brings us to the concept of the material-independent and size-independent tractor beam. Indeed, a tractor beam should only allow beam characteristics which achieve or prohibit the pulling effect, rather than the beam needs to "negotiate" with the object to see if it can be called a tractor beam. In fact, it is not likely to achieve a perfectly universal tractor beam pulling everything, but it is still very meaningful that the size and refractive index of the object can be chosen in a wide and continuous range. As can be seen in Fig. 2, for an object of a specified size, it is always possible to manipulate the force to be a pulling force, even though the permittivity varies within a very wide range. The region may extend to large values of permittivity, which confirms our conclusion that the high permittivity will induce the large Mie scattering coefficient $b_{1}$ in 
Fig. 2(b). It is, however, not the case for the acoustic tractor beam, which will be shown in the following. The size independence can be even robust within a wide range of $k_{0} R$ from Rayleigh to larger particles at a given value of material parameters, as shown in Fig. 3.

The pulling force effect cannot exist when the electric and magnetic polarizabilities are incomparable. However, at $\alpha_{e}=\alpha_{m}$ the optical force $\left\langle F_{z}\right\rangle \sim \beta-1+4(2 \beta-1) y^{2}$ is always less than zero when $\beta<1 / 2$ (here, $y^{2}$ is just a positive quantity). Figure 2(b) indeed reveals that $\left\langle F_{z}\right\rangle<0$ arises for close $\alpha_{e}$ and $\alpha_{m}$. The shaded region of the negative force in Fig. 2(b) covers the region around the violet line of $\alpha_{e}=\alpha_{m}$ (or $a_{1}=b_{1}$ ). This line can be approximated by a hyperbola so that dipole particles become optically pulled for $\sqrt{\varepsilon} k_{0} R$ approximately within 2.5-3.

Absorption does not violate the concept of independent tractor beams. As expected, absorption only shrinks the parameter band of the pulling force and reduces the magnitude of the negative force, but the band is still large even at the absorption level of $\operatorname{Im}(\varepsilon)=0.1$ [Fig. 2(c)]. A further increase of absorption eliminates the pulling for small-Re $(\varepsilon)$ particles.

In contrast to absorption, the magnetic response of a bead makes the pulling force easier. Although the unshaded region of the valid dipole approximation shrinks, the presence of the permeability enlarges the parameter band of the pulling force. For small dielectric permittivities, $\left\langle F_{z}\right\rangle$ becomes insensitive to the particle radius, as the bottom part of Fig. 3 shows. Figure 3 clearly illustrates the improvement of the pulling optical force due to the magnetic properties of the material. Thus, magnetic permeability $\mu$ maintains the concept of the material-independent and size-independent tractor beam.

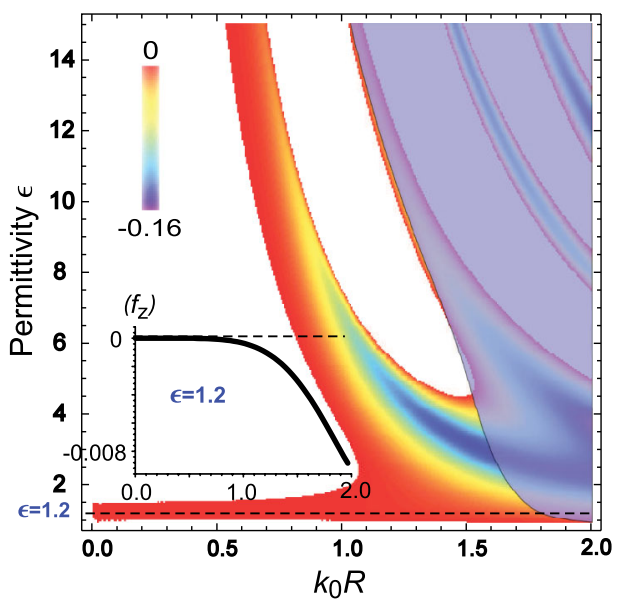

FIG. 3 (color online). The optical pulling force $\left\langle f_{z}\right\rangle=$ $\left\langle F_{z}\right\rangle k^{2} /\left|c_{1}\right|^{2}$ for magnetic particles $(\mu=1.2)$. Parameters: $m=1, \alpha=70^{\circ}, c_{2} / c_{1}=i$. In the inset, the force $\left\langle f_{z}\right\rangle$ vs $k_{0} R$ (beyond the dipole approximation) at $\varepsilon=1.2$ is shown.
Actually, further investigation reveals that dipole objects bear the universally necessary condition for an electromagnetic beam to be a tractor beam. This condition is independent of the material parameters or size of the dipole bead but only imposes a limitation on the longitudinal wave number $k_{z}=k_{0} \beta$ (or cone angle $\alpha$ ). Since the cross product $P_{z}=\mathbf{e}_{z}(\mathbf{E} \times \mathbf{H})$ cannot be greater than the product of the absolute values of the constituent vectors $|\mathbf{E}||\mathbf{H}|$, the pulling optical force $\left\langle F_{z}\right\rangle<0$ requires [see Eq. (3)]

$$
\beta<\frac{k_{0}^{3} \operatorname{Re}\left(\alpha_{e} \alpha_{m}^{*} P_{z}\right)}{3 \sqrt{\operatorname{Im}\left(\alpha_{e}\right) \operatorname{Im}\left(\alpha_{m}\right)}|\mathbf{E}||\mathbf{H}|}<\frac{k_{0}^{3} \operatorname{Re}\left(\alpha_{e} \alpha_{m}^{*}\right)}{3 \sqrt{\operatorname{Im}\left(\alpha_{e}\right) \operatorname{Im}\left(\alpha_{m}\right)}} .
$$

Polarizabilities of the nonabsorptive spheres can be represented as $\alpha_{e, m}=\alpha_{e, m}^{(0)} /\left(1-i(2 / 3) k_{0}^{3} \alpha_{e, m}^{(0)}\right)$, where $\alpha_{e, m}^{(0)}$ are real-valued quantities. Then Eq. (4) takes the form

$$
\beta<\frac{1}{2} \text {. }
$$

This universal condition can be rewritten in terms of the cone angles as $\alpha>60^{\circ}$. Thus, angle $\alpha=60^{\circ}$ is the minimal cone angle, failing which it is not possible to have a pulling force for the passive dipole particles [19].

The concept of independent tractor beams is the consequence of the wave physics and, therefore, may be observed in acoustics. For a zeroth-order nonparaxial acoustic Bessel beam (cone angle $\alpha$ ), the acoustic pulling force $F_{z}=\pi R^{2} I_{0} Y_{P} /\left(c_{0} \cos \alpha\right)$ acting on a sphere of radius $R$ can be presented in terms of the dimensionless function $Y_{P}$, where $c_{0}$ is the sound speed of the ambient fluid, and $I_{0}$ is the acoustic intensity [8]. The scattered function of the velocity potential $\psi_{\mathrm{sc}}=\sum_{n=0}^{\infty} d_{n} \phi^{n}$ is reduced to the sum of monopole $(n=0)$ and dipole $(n=1)$ terms for a dipole bead, where $d_{n}$ defines the strength of multipolelike Mie coefficients in optics. For the dipole approximation, we need to impose small $d_{2}$, compared to $d_{0}$ and $d_{1}$. The monopole-dipole interaction originates the pulling force, analogous to the similar phenomenon (interaction of electric and magnetic dipoles) in electrodynamics discussed above.

In spite of the evident similarity of the acoustic and optical forces, there is a substantial difference. The acoustic pulling force in the dipole approximation cannot be achieved within a large range of $\lambda$, i.e., the ratio of densities of the drop and surrounding fluid. Introducing the condition of the validity of the dipole approximation $\left|d_{2}\right| / \sqrt{\left|d_{0} d_{1}\right|}<0.2$, we get the limitation $\lambda<1.3$ (Fig. 4). At the same time, there are realistic materials allowing the negative force for $\lambda$ 's in this range (see example of a benzene particle in water in Fig. 4). When $\lambda$ is between 1.2 and 1.3 , the pulling force does not depend on the particle's radius $k_{0} R$ in the wide range, including small values. Such behavior corresponds to parameters $\lambda$ and $\sigma$ satisfying equation $1+2 \lambda=3 \lambda^{2} \sigma^{2}$, where $\sigma$ is the ratio of the drop and surrounding fluid sound speeds (see Refs. $[8,20])$. Therefore, the density ratio $\lambda$ provides 


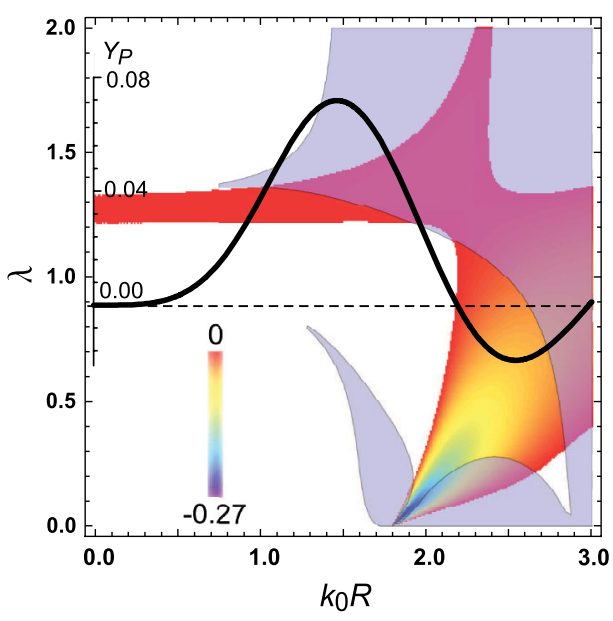

FIG. 4 (color online). Dimensionless pulling acoustic force $Y_{P}<0$ in the dipole approximation versus density ratio $\lambda$ and dimensionless sphere radius $k_{0} R$ for fluid sound speed ratio $\sigma=0.861$ and Bessel beam's cone angle $\alpha=70^{\circ}$. Black curve in the inset corresponds to $Y_{P}$ for a benzene sphere in water $(\lambda=0.874)$ [8]. Shaded region indicates a strong enough quadrupole term using $\left|d_{2}\right| / \sqrt{\left|d_{0} d_{1}\right|}>0.2$.

size-independent rather than material-independent acoustic traction.

In conclusion, we have revealed and demonstrated the novel concept of material-independent and sizeindependent optical pulling forces and explained the origin of universal optical traction. As a general schematic to guide the realization of practical universal tractor beams, a necessary condition is proposed on the nonparaxial angle for Bessel lasers. It is important that the articulated properties do not vanish for absorptive particles. Distinctions of independent tractor beams in optics and acoustics have been discussed within the dipole approximation, in which the acoustic tractor beam only exhibits the size independence. Nevertheless, the material-independent acoustic traction can be expected beyond the dipole approximation. Even if the Bessel beam cannot be ideally nondiffractive experimentally, it is still a long-range propagating wave and the pulling force will work over the long distance. The large numerical aperture (cone angle $\alpha$ ) is the major challenge in experiments, which may be enabled by future development of advanced optics. Hence, these reported findings are believed to be useful in powering the research of tractor beams and, more importantly, to be able to provide guidelines for future experimental verification of universal optical tractor beams.
C.W.Q. acknowledges financial support from Grant No. R-263-000-678-133 of the National University of Singapore. A. N. and A. L. are grateful for partial financial support from the Danish Research Council for Technology and Production Sciences via the THz COW project.

*eleqc@nus.edu.sg

[1] P. T. Korda, M. B. Taylor, and D. G. Grier, Phys. Rev. Lett. 89, 128301 (2002); Y. Roichman, V. Wong, and D. G. Grier, Phys. Rev. E 75, 011407 (2007).

[2] A. Ashkin, J. M. Dziedzic, J. E. Bjorkholm, and S. Chu, Opt. Lett. 11, 288 (1986); D. G. Grier, Nature (London) 424, 810 (2003).

[3] M. Li, W. H. P. Pernice, and H. X. Tang, Phys. Rev. Lett. 103, 223901 (2009).

[4] S. Sukhov and A. Dogariu, Opt. Lett. 35, 3847 (2010).

[5] S. H. Lee, Y. Roichman, and D. G. Grier, Opt. Express 18, 6988 (2010).

[6] E. F. Nichols and G. F. Hull, Phys. Rev. 13, 307 (1901); M. Kerker and D. D. Cooke, J. Opt. Soc. Am. 72, 1267 (1982); M. Kerker, Am. Sci. 62, 92 (1974).

[7] A. Novitsky, C. W. Qiu, and H. Wang, Phys. Rev. Lett. 107, 203601 (2011).

[8] P. L. Marston, J. Acoust. Soc. Am. 120, 3518 (2006).

[9] J. Durnin, J. Opt. Soc. Am. A 4, 651 (1987).

[10] T. M. Grzegorczyk, B. A. Kemp, and J. A. Kong, Phys. Rev. Lett. 96, 113903 (2006).

[11] B. A. Kemp, T. M. Grzegorczyk, and J. A. Kong, Phys. Rev. Lett. 97, 133902 (2006).

[12] J. Chen, J. Ng, Z. Lin, and C. T. Chan, Nature Photon. 5, 531 (2011).

[13] S. Sukhov and A. Dogariu, Phys. Rev. Lett. 107, 203602 (2011).

[14] C.F. Bohren and D. R. Huffman, Absorption and Scattering of Light by Small Particles (John Wiley \& Sons, New York, 1998).

[15] A. Garcia-Etxarri, R. Gomez-Medina, L. S. Froufe-Perez, C. Lopez, L. Chantada, F. Scheffold, J. Aizpurua, M. Nieto-Vesperinas, and J. J. Saenz, Opt. Express 19, 4815 (2011).

[16] A. V. Novitsky and D. V. Novitsky, J. Opt. Soc. Am. A 24, 2844 (2007).

[17] M. Nieto-Vesperinas, J. J. Saenz, R. Gomez-Medina, and L. Chantada, Opt. Express 18, 11428 (2010).

[18] A. Mizrahi and Y. Fainman, Opt. Lett. 35, 3405 (2010).

[19] See Supplemental Material at http://link.aps.org/ supplemental/10.1103/PhysRevLett.109.023902 for support of the findings on universal necessary condition in Eq. (5).

[20] L. K. Zhang and P. L. Marston, Phys. Rev. E 84, 035601 (2011). 